\title{
Norms of Testimony in Broad Interdisciplinarity: The Case of Quantum Mechanics in Critical Theory
}

\author{
Rasmus Jaksland ${ }^{1}$ (D) \\ Published online: 14 October 2020 \\ (C) The Author(s) 2020
}

\begin{abstract}
While much interdisciplinarity brings together proximate fields, broad interdisciplinarity sees integration between disciplines that are perceived to be non-neighboring. This paper argues that the heterogeneity among disciplines in broad interdisciplinarity calls for stricter epistemic norms of testimony for experts that act as translators between the disciplines than those suggested for intra-scientific testimony. The paper is structured around two case studies: the affective turn in social theorizing and the use of quantum mechanics in critical theory as exemplified by Vicky Kirby's use of work by Karen Barad. These are argued to be instances of broad interdisciplinary borrowing where few translators have joint expertise in both disciplines. For most, therefore, the engagement with for instance the integration between quantum mechanics and critical theory is possible only by the aid of translators. For those without sufficient interactional expertise, however, the epistemic credentials of the translations they inevitably rely upon are inscrutable. Furthermore, any comparison between translations is challenged since translations are argued to be few due to the cognitive divergence between disciplines in broad interdisciplinarity. Consequently, the epistemic integrity of broad interdisciplinarity can only be secured through additional norms of testimony for translators. The paper proposes that (a) all translator's testimony in broad interdisciplinarity must aim to be neutral with respect to disputed issues within the relevant disciplines and (b) any deviation from (a) must be clearly highlighted.
\end{abstract}

Keywords Interdisciplinarity $\cdot$ Expert testimony $\cdot$ Quantum mechanics $\cdot$ Epistemic dependence $\cdot$ Karen Barad $\cdot$ The affective turn

\section{Introduction}

Science has outgrown the lonely polymath. Today, science is dominated by collaborations among scientists each contributing their particular expertise (e.g. Kitcher 1990; Weisberg and Muldoon 2009). Scientists thereby come to depend epistemically on each other's testimony: scientists use in their work propositions for which they have themselves no first

Rasmus Jaksland

rasmus.jaksland@ntnu.no

1 Department of Philosophy and Religious Studies, Faculty of Humanities, NTNU - Norwegian University of Science and Technology, Trondheim, Norway 
order evidence but only the assurance of an expert that the proposition is true. However, not all epistemic dependence is the same. As Susann Wagenknecht (2014) observes, there is an important difference between cases where one has the expertise to obtain this first order evidence, if necessary (translucent epistemic dependence), and cases where one does not have the required expertise to do so (opaque epistemic dependence). By means of a case study - the use of quantum mechanics in critical theory - this paper proposes the relevance of a further subdivision between opaque and very opaque (denoted impenetrable) epistemic dependence; the latter being a type of epistemic dependence that, due to the lack of expertise on the dependent part, can only occur by the mediation of a translator. ${ }^{1}$ It is then in turn proposed that the epistemic conditions in impenetrable epistemic dependence are such that experts providing testimony-the translators - should adhere to additional epistemic norms of assertion as compared to experts in cases of opaque and translucent epistemic dependence.

The case study is an example of broad interdisciplinary borrowing where two cognitively divergent disciplines (broad) collaborate in such a way (interdisciplinary) that the findings of one discipline are used in the other (borrowing). More precisely, the case study shows how critical theorist Vicky Kirby (2011) informs her discussion of the nature/culture dualism by the findings of quantum mechanics as reported by Karen Barad (2007). Barad thereby acts as a translator of quantum mechanics for Kirby, presumably because Kirby does not have the required expertise to borrow directly from the research literature on (the interpretation of) quantum mechanics. Observing that similar circumstances obtain in the affective turn where neuroscience is used in social theorizing, the paper argues that these patterns of indirect borrowing through translations are likely to be widespread in broad interdisciplinarity where multi-disciplinary expertise is particularly hard to come by. The problem is that translations, in the absence of multi-disciplinary expertise, attain an epistemic inscrutability (to be further specified below) whereby the epistemic dependence on translators becomes impenetrable. A concrete consequence of this is that Kirby echoes Barad's idiosyncratic interpretation of quantum mechanics as though it were the undisputed facts about quantum mechanics.

Taking inspiration from inter-language translations, two initiatives are considered that might lessen the effect of the precarious epistemic circumstance that indirect borrowing through translators provides for: The comparison of multiple translations and requiring that translations are properly annotated. The former, however, proves to be problematic due to the scarcity of translators and translations. To secure the epistemic integrity of broad interdisciplinarity where impenetrable epistemic dependence occurs, it is therefore argued that its deficiencies must be countered by additional norms of assertion for translators' testimony (in broad interdisciplinarity): (a) all testimony must aim to be neutral with respect to disputed issues within the relevant disciplines and (b) any deviation from (a) must be clearly highlighted. In other words, the translations should be properly annotated.

In the following, Sect. 2 introduces broad interdisciplinary borrowing through two examples: One is the affective turn which has already been studied as an interdisciplinary phenomenon. The other is Barad's works on the interpretation of quantum mechanicsespecially Meeting the Universe Halfway (2007) — and how it is used by Kirby in her book Quantum Anthropologies (2011). Section 3 defines the notion of impenetrable epistemic

\footnotetext{
1 Throughout this paper, the terms 'translation', 'translator', 'mediation', and 'intermediate' are used in their everyday sense following Collins et al. (2007). They are therefore not meant to carry the specific technical meaning associated with them in actor network theory.
} 
dependence, argues that it is exemplified Kirby's use of Barad, and that impenetrable epistemic dependence can be expected to be frequent in other broad interdisciplinary borrowings. Section 4 explores the epistemic risks involved in impenetrable epistemic dependence; especially the way it enables the uncritical transmission of questionable aspects of a translators' testimony into entire interdisciplinary (sub)fields. The two ways to manage this epistemic risk is discussed in Sect. 5 which concludes that the comparison of translations is challenged in broad interdisciplinarity. Instead, Sect. 6 proposes that the epistemic risks associated with broad interdisciplinary borrowing must be countered by the additional norms of assertion (a) and (b) for translators' testimony. These norms are subsequently discussed in the light of the case study before a conclusion follows.

This exploration of the epistemic circumstances in broad interdisciplinarity and the proposal of additional norms of testimony are not intended as a criticism of the work of Barad, Kirby or any other of the researchers mentioned. In particular, this paper involves no assessment of this work and its value to critical theory. What the paper discloses is rather a pattern of epistemic dependence whereby idiosyncratic interpretations, in the case of quantum mechanics, are at risk of being transmitted without the knowledge of the recipient. The paper aims to argue how to avoid this pattern. In arguing that Kirby's use of Barad's work exemplifies this pattern, the paper finds that Barad's Meeting the Universe Halfway does not satisfy the proposed norms of testimony even though the epistemic circumstances, by the argument of this paper, calls for their adherence. This should not be seen as an attempt to cast Barad as dishonest but should rather be perceived as a note of caution for broad interdisciplinarity moving forward.

\section{Broad Interdisciplinarity}

In integrated interdisciplinarity, "the concepts and insights of one discipline contribute to the problems and theories of another" (Klein 2010, 20). Often, this follows the pattern of borrowing whereby the elements of one discipline are used to inform the concerns of another (Klein 1990, Ch. 5). Paradigmatic examples of borrowing include the use of thermodynamics to study chemical reactions, Darwin's use of geology in the development of the theory of evolution, and the use of psychoanalysis in folklore studies (Klein 1990, 85-87). Borrowing can take place between all disciplines, but the interest here is borrowings in cases of broad interdisciplinarity that "occurs between disciplines with little or no compatibility, such as science and humanities" (Klein 2010, 18). Broad interdisciplinarity is contrasted with narrow interdisciplinarity which "occurs between disciplines with compatible methods, paradigms, and epistemologies, such as history and literature [...] (Klein 2010, 18) ${ }^{2}$ The defining characteristic of broad interdisciplinarity can in this sense be summarized by the wide "cognitive divergence" between the collaborating disciplines (Andersen 2016, 7).

The so-called 'affective turn' (e.g. Clough and Halley 2007) in social theorizing is a recent example of broad interdisciplinarity with borrowing that has received some scrutiny as an instance of interdisciplinarity. Papoulias and Callard (2010,30-31), in particular, have explored "the consequences of cultural theory's strange borrowings from neuroscience and developmental psychology" $(2010,29)$ and found that they are "used in order to

${ }^{2}$ For a further discussion of narrow and broad interdisciplinarity, see Newell (1998). 
ground certain claims about affect within cultural theory" (Papoulias and Callard 2010, 29). The findings of these sciences are used to buttress the modifications to social theory advocated by the turn to affect. Somewhat polemically, Fitzgerald and Callard (2014) call this mode of interdisciplinarity "ebullience"; a mode of interdisciplinarity where researchers in the humanities and social sciences "assign to the natural and experimental sciences the task of generating the findings that will confirm, verify and/or reveal the theoretical insights of cultural and social theory" (Fitzgerald and Callard 2014, 12-13). It is precisely this ebullience mode of interdisciplinarity that is of interest here, and the mode, as shall be argued, that Barad's and Kirby's work also exemplifies though with neuroscience replaced by quantum mechanics. However, it is important to emphasize that the present analysis of the epistemic circumstances in broad interdisciplinarity is not limited to instances where the direction of borrowing is from the natural sciences to the humanities and social sciences. Rather, the relevance of the analysis only requires that the borrowing, in the manner of ebullience, is of the findings of a discipline and that the involved disciplines have wide cognitive divergence. The argument should therefore equally apply to for instance the proposed uses of literary theory in cognitive science (e.g. Turner 1996), though this case will not be pursued further here.

Barad's Meeting the Universe Halfway: Quantum Physics and the Entanglement of Matter and Meaning (2007) is an immediate candidate for broad interdisciplinarity in forming a meeting - as the title already suggests - of quantum mechanics and the contested dualism of matter and meaning; the latter being a common theme in critical theory broadly construed and in new materialism in particular (see Dolphijn and Van der Tuin 2012). In the book, Barad develops the metaphysical ${ }^{3}$ framework of what she calls agential realism ${ }^{4}$ based on an ontological reading of Niels Bohr's interpretation of quantum mechanics and defends this framework through detailed accounts of quantum experiments while providing numerous references to the physics literature on quantum mechanics. ${ }^{5}$ Despite this origin of Barad's work, Fairchild and Taylor find that "her influence in the fields of new materialism, new material feminism, science studies, queer studies, and posthumanism has been profound" (2019). Neither science studies nor feminism are of course strangers to physics in general or quantum mechanics in particular (e.g. Aronowitz 1988; Harding 1986; 1991; Hayles 1984; Keller 1995; Pickering 1984; Plotnitsky 1994). These works, however, can generally be characterized as cultural studies of physics and quantum mechanics; in the taxonomy of Helene Götschel (developed specifically for the entanglements of gender and physics), this type of approach covers "Human actors in physics", "Work place cultures in physics", and "Knowledge production in physics" (2011, 67). While Götschel argues that Barad is also concerned with the latter, she finds that Barad's work is so far unique in "the inclusion of approaches coming from physics into the development of new theoretical and methodological concepts" $(2011,67)$. Arguably, also Donna Haraway's development

\footnotetext{
3 Ethic-epistem-ontological in Barad's own terms $(2007,90)$.

${ }^{4}$ Giving a full account of Barad's agential realism with all its subtleties is well beyond the scope of this paper. The interested reader is advised to consult Barad's own writings on the subject; especially Meeting the Universe Halfway (2007). Those interested in an exposition of agential realism as a feminist philosophy of physics can consult Harrell (2016, Sect. 2.3.3). Agential realism in the broader context of feminist philosophy of science and its "moving between naturalized descriptions of science and constructive reformulations of scientific norms" is further discussed by Richardson (2010, 349). Finally, agential realism and its relation to naturalism in general is explored in Rouse (2004).

5 Citing articles in renowned journals such as Nature, Physical Review Letters, Physical Letters, Physical Review D, and Physical Review A, among others (Barad 2007, References).
} 
of the methodological concept of 'diffraction' could fall under this description by its origin in (quantum) optics. However, whereas optics is a metaphor or analogy for the workings of Haraway's notion of diffraction, this is not the case for quantum mechanics in Barad's work:

I am not interested in drawing analogies between particles and people, the micro and the macro, the scientific and the social, nature and culture; rather, I am interested in understanding the epistemological and ontological issues that quantum physics forces us to confront (Barad 2007, 24). ${ }^{6}$

Agential realism captures the epistemological and ontological consequences of quantum mechanics which we are forced to confront in all theorizing. The findings of quantum mechanics thereby serve a justificatory purpose for the interventions that agential realism, on Barad's own initiative, makes in social theorizing. In this respect, the role of quantum mechanics in agential realism resembles that of neuroscience in the affective turn where "[n]euroscience emerges [...] as a kind of mainspring of cultural theory, capable of accounting for its method, if not its very existence" (Papoulias and Callard 2010, 38). Barad's work-like the interdisciplinary work of the affective turn-is, in other words, an instance of ebullience.

By the title, Kirby's book Quantum Anthropologies: Life at Large seems to engage in a similar program. Though quantum mechanics plays a less central role in the book than might be anticipated from the title, Kirby describes how "[t]he quantum problematic will be taken up briefly in chapter 4 , and in more extended discussion in chapter 6" (Kirby $2011,146)$. In chapter 4 , she invokes certain elements of quantum mechanics as "of crucial importance for my argument" $(2011,76)$ and chapter 6 in turn includes the promised extended discussion exploring the implication of quantum entanglement and so-called quantum eraser experiments. Kirby describes these implications as "in rhythm with my reading of Merleau-Ponty" $(2011,126)$ whereby quantum mechanics promises to corroborate "[a]n intra-ontology such as Merleau-Ponty offers [that] reframes questions of ontology, epistemology, ethics, and science by radically recasting the anthropological" (Kirby 2011, 136). ${ }^{7}$ In this way, also Kirby borrows from the findings of quantum mechanics to pursue issues in critical theory whereby her Quantum Anthropologies qualifies as broad, ebullient interdisciplinarity.

However, except for a few honorable mentions of canonical quantum mechanics publications (Einstein et al. 1935; Bell 1964; Aspect et al. 1982)—all suggestively absent from the bibliography of the book-Kirby does not engage directly with the physics literature on quantum mechanics. Instead, Kirby's primary (and more or less sole) reference on quantum

\footnotetext{
6 This is also observed by Hollin et al., who writes: "Quantum physics, for Barad, is resolutely not a metaphor but, rather, underpins agential realism's articulation of how the material world is brought into being. The material grounding in physics is significant because it promises a stability and reality that can be pressed into service as support for an array of political projects" (2017, 935-936; see also Pinch 2011, $440)$. Indeed, Barad $(2007,6,7,18,24,70,88)$ frequently dismisses any role and even the very legitimacy of regarding the use of quantum mechanics as analogical reasoning and argumentation: "To ask whether it is not suspect to apply arguments made specifically for microscopic entities to the macroscopic world is, in this case, to mistake the approach as analogical" (Barad 2007, 70).

7 The details of Kirby's thesis are not of importance here, and we will therefore refrain from a detailed account.
} 
mechanics in Quantum Anthropologies is Barad (2007). ${ }^{8}$ When borrowing from quantum mechanics, Kirby relies on Barad's authority as signified by remarks of the type "as Karen Barad makes clear, and this point is of crucial importance for my argument, if the different parts of the experiment - the object under investigation, the inquiring scientist, and the apparatus, are 'entangled', then this does not mean that there are three 'entities' interacting with each other" (Kirby 2011, 76) and "[t]he context is Karen Barad's exposition of why our sense of a reality made up of individual objects [...] fails to appreciate that we are dealing with 'phenomena' whose very being is always and only an articulation of entanglement" (Kirby 2011, 126). Both Kirby and Barad use or "borrow" elements of quantum mechanics in the work on themes usually regarded as situated within critical theory. ${ }^{9}$ However, Kirby's interdisciplinary engagement is mediated by Barad's testimony, whereby Barad serves as a translator-and Meeting the Universe Halfway as a translation-of the findings of quantum mechanics for Kirby; a pattern of dependence that will be crucial for the discussion in the following sections. This is not to say that all users of Barad's work display this pattern. For many of these, the quantum origin of agential realism has no justificatory significance (though it might serve as a useful analogy in parallel to Haraway's use of optics). When discussing the epistemic patterns that result from the use of Barad's work as a translation, these only apply to users of agential realism, such as Kirby, where it is important that agential realism actually conveys what quantum mechanics tells us about the world. ${ }^{10}$ This use of Barad's work as a translation is exactly why the accuracy of Barad's account of quantum mechanics is important; a question we will return to in Sect. 4.

The role of translators is also implicit in Papoulias and Callard's account of the affective turn, when they, speaking of biology and neuroscience, stress "that these disciplines are not taken up tout court: rather, it is a select number of scientists who find favour" and speculate that those taken up are often scientists "attempting to construct a composite language that crosses very different domains of analysis" (Papoulias and Callard 2010, 33). These same scientists-Damasio (2003) being an example_-are also described as among "a number of neuroscientists [that] are intensely interested in, and contributors to, philosophical debates concerning the implications of their scientific research" (Papoulias and Callard 2010, 33). As such, Damasio among others seems to serve as a translator for the affective turn in similarity with Barad's role in Kirby's work. Along the same line, Fitzgerald and Callard remark that "many ebullient engagements with the neurosciences from humanists and

\footnotetext{
8 Two footnotes also refer to Nadeau and Kafatos' The Non-Local Universe: The New Physics and Matters of the Mind (2001), but all my remarks_including those concerning the likely audience-made about Barad (2007) apply equally well to Nadeau and Kafatos.

9 Though Barad intends her work as "a legitimate interpretation" of quantum mechanics, and Kirby finds that her rethinking of the nature/culture distinction should be of significance to science — presumably quantum mechanics among them-interactions are not realized as signified by Barad's absence in the philosophy of physics literature. The asymmetric notion of 'borrowing' seems therefore to better capture the details of the case study compared to the more symmetric 'integrative interdisciplinarity'.

${ }^{10}$ New materialism, for instance, is rich in references that are indicative of a justificatory role for the quantum origin of agential realism. An example is a discussion of the integration of ontology and epistemology and the pervasiveness of agency in new materialism which is followed by the remark that "Barad provides a particularly compelling basis for such a view through her 'intra-active' account of the 'measurement problem' in quantum physics" (Gamble et al. 2019, 122). See also the following: "Barad develops her problematization of representational thinking via a detailed account of the scientific apparatus through which reality is observed and measured in quantum physics" (Coleman 2014, 35) and "Karen Barad's (2012) recent discussions with quantum field theory assist greatly in this endeavour to reconsider negativity" (Hinton 2017, 234).
} 
social scientists barely stray further than scientists' 'crossover' publications for lay audiences" $(2014,11)$. In other words, the use of translations goes beyond quantum mechanics in critical theory and seems to be a more general phenomenon in broad interdisciplinarity.

While Papoulias and Callard present this use of selected scientists' crossover publications as a concern relating to the affective turn, this paper will argue that the epistemic circumstances in broad interdisciplinarity are often such that many-in particular those with mono-disciplinary training - must rely on translators in ebullient engagements; though this translation can be into the language of another specialized discipline-which is arguably the case for Barad-and not into that of the lay audience. This, however, does not lesson the relevance of the worries that Papoulias and Callard raise about this use of "crossover publications". Referring to the scientists taken up by the affective turn, Papoulias and Callard find it "important to bear in mind that the research of many of those figures is subject within the natural sciences to significant debate and contestation" $(2010,33)$ and that their work, in some cases, is old by the standard of neuroscience and thereby potentially outdated $(2010,42) .{ }^{11}$ When ebullient engagements rely on the translations of few researchers, debate and (recent) criticism are at risk of not being properly mediated. Furthermore, Papoulias and Callard argue that these uses of neuroscience often form "a strange and partial (mis)translation of complex scientific models into the epistemologically distinct space of the humanities and social sciences $(2010,31)$. Section 4 will argue that these issues are shared by the epistemological space surrounding Barad's work indicating that they are not unique to the affective turn. ${ }^{12}$ Furthermore, this paper finds that the epistemic risks associated with mistranslations and the mediation of contested content (among others) are generally amplified in broad interdisciplinarity since many must inevitably rely on translators. This, ultimately, is the reason why the present paper finds that translators must abide by stricter norms of assertion than those observed within disciplines. Where Papoulias and Callard end their analysis raising these problems, this paper begins by arguing that they are often inevitable in broad interdisciplinarity and therefore calls for general measures to lessen their effect; though, as shall also be argued, the epistemic circumstances in broad interdisciplinarity ultimately render these risks ineliminable. This analysis will also provide an epistemic framework in which to assess the epistemic risk of such interdisciplinary borrowings including (but not restricted to) the affective turn as well as the borrowings from quantum mechanics in critical theory. ${ }^{13}$

\footnotetext{
${ }^{11}$ Fitzgerald and Callard air a similar worry giving the example of Catherine Malabou's (e.g. 2012) works on neuroscience and subjectivity which they find to "demonstrate limited engagement with peer-reviewed scientific publications, with internal criticisms of Damasio, and with histories of science" (Fitzgerald and Callard 2014, 12).

${ }^{12}$ Indeed, they arguably fall under the general hazards of interdisciplinary borrowing already indexed by following Klein (1990, Ch. 5) which will be discussed in more detail in Sect. 4.

${ }^{13}$ With the focus of the present paper being the epistemic circumstances in broad interdisciplinarity, the reader is referred to Papoulias and Callard's work for a discussion of the interesting movements between foundational and afoundational approaches in the affective turn that is also found among the users of Barad's work. Similarly, the present paper merely establishes what epistemic pattern the borrowings from science provide for, whereas Papoulias and Callard is a better starting place for those interested in why science is invoked; an important question given Dunk's observation that "recently, in many areas of social and philosophical research, the "quantum" label has become extremely desirable" $(2019,1)$. Related to this question, Julie Klein $(1990,86)$ finds that the disciplines borrowed from tend to be more structured and scientific than the borrowing discipline: "the most successful theories tend to become 'obsessive paradigms' for other fields of knowledge" (Klein 1990, 86; referring to Hadač 1977).
} 
Before proceeding to this analysis, a remark is in order about the framing of the meeting between quantum mechanics and critical theory as broad, ebullient interdisciplinarity whereby the account places itself within what Fitzgerald and Callard call the "regime of the inter-" $(2014,15)$. In the 'regime of the inter-' - and thus throughout the present paper-the disciplinary boundaries and disciplines' methods and subject matters (including their overlap) are assumed to be fixed. Fitzgerald and Callard, however, recommend a different approach to interdisciplinarity—what they denote "experimental entanglement" "where there are neither neatly bordered disciplines nor any clear dispensation regarding which 'objects' of study are appropriate for each" (Fitzgerald and Callard 2014, 16). This is mentioned since Fitzgerald and Callard call upon Barad's agential realism as the ontological and epistemological framework for experimental entanglements. And they are only one example, including in Barad's own work, where agential realism features in a rethinking of the knowledge producing practices of science including the disciplinary boundaries. Approaching Barad's work assuming the 'regime of the inter-' might therefore seem inappropriate. However, as documented above, Barad does very clearly ground agential realism in the findings of quantum mechanics. As remarked by Trevor Pinch: "I find it deeply puzzling that Barad can call for a more situated account of science and at the same time fail to situate the very part of science she is talking about, while drawing in a realist mode upon experiments to support her position" (2011, 439; see also Willey 2016). Despite Barad's (2011) reply to Pinch, these two components in Barad's writing have in my view not been convincingly reconciled, and the subsequent analysis will therefore take the risk of dealing with only one them, ebullience, while largely ignoring the experimental entanglement. ${ }^{14}$

\section{Epistemic Dependence in Broad Interdisciplinarity}

In epistemological terms, Kirby's reliance on Barad's translation of the findings of quantum mechanics makes Kirby epistemically dependent on Barad's testimony. Epistemic dependence occurs, according to John Hardwig (1985), when we believe in a proposition, $p$, by the appeal to the authority of an expert, rather than by appeal to first order evidence for $p$, where first order evidence is "being defined roughly as anything that counts toward establishing the truth of $p$ " (Hardwig 1985, 336). Arguably, the rich epistemic space of quantum mechanics forms a rather complex chain of epistemic dependence that ends at numerous experimentalists and theoreticians each contributing the various pieces of first order evidence relevant for each proposition within this space. However, what is of interest here is the difference between the character of Barad's and Kirby's epistemic dependence on the epistemic space of quantum mechanics: Barad's epistemic dependence is distributed between the many researchers who have been involved in the scientific labor reported in the numerous physics publications that Barad cites. In contrast, Kirby depends on Barad's testimony for all aspects of Quantum Anthropologies (2011) that relate to quantum mechanics. While Kirby ultimately relies on the same quantum mechanical chain of epistemic dependence as Barad, Kirby has Barad as an intermediate link. In the terminology of the previous section, Barad acts as a translator for Kirby. Kirby is epistemically dependent

\footnotetext{
14 Thank you to an anonymous reviewer of Journal for General Philosophy of Science for pressing me on this point.
} 
on Barad and, as we shall argue, in an impenetrable way such that she must simply rely on Barad's testimony. ${ }^{15}$

This difference in the pattern of epistemic dependence between Kirby and Barad might be explained by a difference in their interactional expertise which Collins and Evans describe as "the ability [actual or latent] to interact with other people, to talk smoothly about the domain [...], to reflect upon their subject matter so as to articulate their findings or judgement, and sometimes to translate the expertise of one domain into the language of another [...]" (Collins and Evans 2007, 37). According to Collins and Evans, disciplines can be viewed as Wittgensteinian 'forms of life', and interactional expertise involves the linguistic socialization into a discipline's form of life. Using an image we shall return to in Sect. 4, interactional expertise is "the ability to master the language of a specialist domain" (Collins and Evans 2007, 14). Interactional expertise, however, is in this sense only partial since it does not involve fluency in the entire culture: Interactional expertise "provides no access to the other parties material culture except in so far as that material culture is represented in discourse" (Collins et al. 2007, 661). Full fluency would require "contributory expertise" where someone has the expertise to advance the discipline by being immersed in its form of life. Within the field of quantum mechanics, Barad possesses interactional, and even in some cases contributory expertise. ${ }^{16}$ As signified by Barad's numerous references to the physics literature, she is fluent in the language of the physics community and discusses both the theoretical and experimental findings of quantum mechanics with reference to the physics publications where these findings are reported and discussed; what we shall denote 'the original context' of these findings. ${ }^{17}$ Kirby, on her part, makes no references to the original context of quantum mechanics. Rather, Barad's work informs Kirby's discussions of quantum mechanics; something that at least indicates that Kirby does not have sufficient interactional expertise to engage directly with quantum mechanics in its original context.

Interactional expertise typically provides for a "fractionated trading zone" where each of the collaborating disciplines retain their distinct cultures (Collins et al. 2007, Sect. 2.3); thus resembling the circumstances of ebullient interdisciplinarity. It is fractionated since only fractions of the cultures meet and thereby, the cultures remain separate. This is why interactional expertise has a central role:

it is precisely the continuing discontinuity between the cultures that enables the individual with interactional expertise, and who thus has a mastery of both languages, to maintain their special role. For example, interpreters can do their job via interactional expertise, going backward and forward between the two groups only so long as the two groups want to communicate but are unable to do so (Collins et al. 2007, 662).

\footnotetext{
15 A similar pattern of epistemic dependence is for instance displayed by Norah Bowman (2019) who develops "Quantum Models for Decolonizing Canadian State Onto-Epistemology" based on Alexander Wendt's (2015) authority on the implications of quantum mechanics for social sciences (though a couple of references are also made to Barad (2007)). Many of the worries raised about Barad's work and role as a translator, also apply to Wendt.

16 As signified by her publications of well renowned physics journals (Barad 1984; 1988).

17 Thereby, 'the original context' is meant to denote research literature that the findings are native to as opposed to the reporting of these findings in other resources such as Barad's (2007) reporting on quantum mechanics. While Barad (2007) is not considered the original context for findings in quantum mechanics, it might be the original context for other findings such as the (alleged) relation between quantum mechanics and critical theory.
} 
Those with interactional expertise can take on the role as translators and mediate between the collaborating disciplines; a framework that will serve as template for the interdisciplinary mode of translators explored here. However, the present emphasis is not so much on collaborations but on borrowing, i.e. a one-way communication whereby the translators go from one group to the other, but not necessarily the other way around. ${ }^{18}$

In broad interdisciplinarity where there is wide cognitive divergence between the collaborating disciplines, translators will take a particularly central role. Describing broad interdisciplinarity as "peer-different collaborations", Paul Thagaard argues that in such collaborations "cross-disciplinary education will be required for people from different fields to be able to work together productively" $(1997,253)$. For those with only mono-disciplinary training - and generally those with insufficient interactional expertise-any engagement in research on for instance the integration between quantum mechanics and critical theory will therefore typically rely on the mediation of translators who have joint expertise in both disciplines (Klein 1990, 93-94). This role of translators resonates well with "the burden of comprehension" (Krishna 1977) in interdisciplinary borrowing which "requires that borrowers acquire at least a basic understanding of how something is used in its original context" (Klein 1990, 88). Without this understanding, without partial interactional expertise, researchers of the borrowing discipline will find it difficult to directly obtain even acquaintance with the results or conclusions of the discipline borrowed from.

Thagaard finds that "[p]eer-different collaborators are exceptionally epistemically dependent on their coworkers, they typically lack the skill to validate work done in a different field" $(1997,254)$. However, there seem to be important differences as to how epistemically dependent collaborators are. This was already indicated above by the difference in Kirby's and Barad's relation to the quantum chain of epistemic dependence. In more general terms, Wagenknecht proposes a distinction between opaque and translucent epistemic dependence: "A scientist is opaquely dependent upon a colleague's labor, if she does not possess the expertise necessary to independently carry out, and to profoundly assess, the piece of scientific labor her colleague is contributing" $(2014,483)$. This opaque epistemic dependence is contrasted with translucent epistemic dependence where the scientist in question does possess the necessary expertise, but for some reason has not exercised this expertise to overcome the epistemic dependence.

According to Wagenknecht, "interactional expertise has the potential to render dependence relations 'less' opaque" (Wagenknecht 2014, 490). However, even Barad's interactional expertise in quantum mechanics is short of proving the expertise to independently carry out all the scientific labor (including experimental work) that she references; what Wagenknecht appears to regard as a necessary condition for translucent epistemic dependence. Still, Barad arguably displays the expertise to (at least partially) assess the quantum mechanical findings she relies upon through an understanding of how these findings are obtained and why they are reliable. In contrast, Kirby (e.g. 2011, 76) merely recounts certain findings of quantum mechanics and refers to Barad's authority for their sanctioning; something that suggests the absence of an independent assessment of the findings.

\footnotetext{
18 This one-directed focus is partly motivated by the absence of actual communication. As Papoulias and Callard remark in the context of the affective turn, it is not "clear what the quality of such a dialogue might be like given that-figuratively, if not literally - there appear to be relatively few neuroscientists in the room interested in participating in those "conversations"' (Papoulias and Callard 2010, 38). Similarly, no physicists and few philosophers of physics have until now shown any interest in agential realism; a theme we shall return to in Sect. 5 .
} 
To better capture this difference between Barad and Kirby, the notion of opaque epistemic dependence adopted here shall be slightly more liberal than Wagenknecht's.

Definition: A researcher is opaquely dependent on a collaborator's labor, if the researcher does not have the expertise, if necessary, to assess the scientific labor through understanding how it was obtained ${ }^{19}$ and by surveying related findings that might question or affirm it.

For brevity, the term 'assess' is adopted to capture this whole condition. In involving two requirements - understanding and surveying - a distinction can be made between content assessment based on understanding of the findings and the weaker survey assessment based on awareness of the absence of contradictions or presence of confirmations of the findings; something that can arguably be achieved without understanding.

Following this more liberal construal, Kirby's epistemic dependence on the quantum mechanics chain of expertise remains opaque, whereas Barad-by virtue of her (greater) interactional expertise - can assess the quantum mechanical findings, and her epistemic dependence on the quantum mechanics chain of expertise therefore qualifies as translucent. However, the difference in their ability to assess the testimony relied upon is not the only distinguishing feature between Barad's and Kirby's epistemic dependence. As already hinted at above, Barad bases her discussions of quantum mechanics directly on the publications in which these findings are first reported-what was denoted 'the original context'whereas Kirby uses an indirect source of testimony not belonging to the original context, namely Barad's work. Apparently, Kirby has insufficient interactional expertise to even acquire the findings of quantum mechanics directly from this original context, let alone subject it to epistemic assessment. Instead, she must resort to Barad's testimony whereby Barad acts as a translator of quantum mechanics for Kirby and other critical theorists that engage in this type of broad interdisciplinarity, but who lack the required interactional expertise to borrow directly from quantum mechanics themselves. Barad is the actual borrower and thus an intermediate in Kirby's epistemic dependence on quantum mechanics.

Interestingly, this is indicative of another dimension of importance in the space of epistemic dependence that is not identified by Wagenknecht and which relates to the expertise to acquire the borrowed findings from the research literature in which these findings are reported and discussed, i.e. from the original context. Where the translucent/opaque distinction maps the expertise to (profoundly) assess evidence for a proposition, $p$, if necessary, this other distinction maps the ability to acquire $p$ directly from its original context, if necessary. Following this, Kirby's epistemic dependence on quantum mechanics is indirect, since she acquires the results of quantum mechanics, not directly from the physics literature, but by means of a translator: Barad. Whereas the physics literature on quantum mechanics - the original context-is inaccessible to Kirby, Barad's Meeting the Universe Halfway serves as a bridge such that Kirby can acquire this knowledge indirectly despite the absence of expertise to acquire it directly. While Barad also depends on the quantum mechanics chain of expertise, she does so directly in that she acquires the findings of quantum mechanics from their original context. We shall map this distinction by the terms direct (Barad) and indirect (Kirby) epistemic dependence. A researcher is directly

\footnotetext{
${ }_{19}$ More precisely, for theoretical work this requires understanding of the reasoning-including the relation between formal steps - of the argument, and for experimental work, it requires the understanding the experimental setup including how the experiment could give the results it did.
} 
dependent on a collaborator's labor, if the researcher possesses the expertise necessary to acquire the results of this labor as it occurs in its original context. A researcher is indirectly dependent if such an expertise is absent, and therefore requires a translation that mediates the content of the scientific labor in question.

\begin{tabular}{lll}
\hline & Translucent & Opaque \\
\hline Direct & $p$ can be acquired from & $p$ can be acquired from, but not assessed in its original context. \\
& $\begin{array}{l}\text { and assessed in its origi- } \\
\text { nal context. }\end{array}$ & \\
Indirect & $\begin{array}{l}\text { Unintelligible: Would } \\
\text { involve ability to assess } \\
\text { but not acquire } p .\end{array}$ & $\begin{array}{c}p \text { cannot be acquired from or assessed in its original context. } \\
\text { Acquaintance only possible by means of translator. }\end{array}$ \\
& & \\
\hline
\end{tabular}

The distinction between direct and indirect epistemic dependence signifies an important subdivision in opaque epistemic dependence. While translucent and indirect epistemic dependence look irreconcilable, opaque epistemic dependence can be both direct and indirect. In direct, opaque epistemic dependence, one can acquire a proposition directly from its original context and this at least leaves in place the preconditions for a survey assessment. Even if one has insufficient interactional expertise for a content assessment, the expertise to acquire a proposition directly should in most cases be sufficient to survey the original context for confirmations, negations or moderations of the acquired proposition. Such a survey assessment can give some justification for one's credence in a proposition that is not based on those providing it as testimony. This still qualifies as opaque epistemic dependence, but the epistemic circumstance is better than in cases of indirect, opaque epistemic dependence. Here, the inability to acquire the proposition directly from its original context precludes such a survey assessment (though it might be conducted on the level of translations as discussed in Sect. 5). One must therefore rely on translators, not only to acquire the proposition, but also in forming a belief about the reliability of the proposition; for instance whether it is contested, outdated, discussed, or generally agreed upon. ${ }^{20}$ For this reason, we shall subsequently reserve the term 'opaque' for the direct, opaque epistemic dependence and denote the indirect form by 'impenetrable epistemic dependence'.

Most instances of epistemic dependence within a discipline are direct or at least trivially indirect; where one has acquired $p$ from handbooks, textbooks, or reviews. In narrow interdisciplinarity, for instance as exemplified by a collaboration between history and literature, the compatibility of methods and paradigms facilitates the required interactional expertise for direct epistemic dependence even for those with mono-disciplinary training; here everyone will have the ability to do the borrowing themselves (though they might not do so out of convenience). By the same reasoning, however, indirect epistemic dependence is more widespread in broad interdisciplinarity; as exemplified by the case study. ${ }^{21}$

\footnotetext{
${ }^{20}$ Goldman's (2001, 93) five sources of argument-based evidence (A-E) reflect this differences between opaque and impenetrable epistemic dependence: only A is challenged in direct, opaque epistemic dependence: trusting an expert's testimony based on the evidence they give for it. However, in impenetrable epistemic dependence (indirect, opaque epistemic dependence) also B, the conclusions of other experts, and $\mathrm{C}$, the approval or disproval of the expert by meta-experts, are challenged. $\mathrm{B}$ and $\mathrm{C}$ require sufficient interactional expertise to survey the community of experts in the original context. This expertise, however, is exactly what is absent when someone's epistemic dependence is impenetrable.

21 Interdisciplinary collaborations often go by personal interaction among scientists from different disciplines. This arguably blurs the notion of 'translator' and therefore the distinction between direct and indirect epistemic dependence. This issue, however, will not be pursued further here.
} 
Consequently, we will often find impenetrable epistemic dependence in broad interdisciplinarity. ${ }^{22}$ This brings translators center stage: Those with insufficient interactional expertise-most often those with only mono-disciplinary training-must inevitably rely on the testimony of translators if they are to engage in broad interdisciplinarity. This is an important discovery, since the next section will argue-again by example of quantum mechanics in critical theory - that impenetrable epistemic dependence with its need of translators provides for a somewhat precarious epistemic circumstance.

\section{Epistemic Risks in Broad Interdisciplinarity}

Following Collins et al.'s (2007) construal of interactional expertise as linguistic socialization, the description of work such as Barad's as translations can be taken quite literally and doing so helps to recognize some (but not all) of the epistemic challenges of impenetrable epistemic dependence. Consider the example of monolinguals who use translations from another language for their academic work. In general terms, monolinguals must rely on the faithfulness of these translations. The lack of expertise in the language of the original entails that they cannot scrutinize the quality of the translation by means of comparison to the original. More particularly, this includes the inability to assess (again by means of such a comparison):

1. where the translator has made translational choices when they inevitably occur and how consequential these choices are.

2. whether the translational choices are idiosyncratic or motivated by a peculiar reading of the original text as a whole.

3. whether certain passages - or the two languages as a whole — do not translate well such that translation distorts the original.

With the relevant linguistic competence, bilinguals can (at varying degree) circumvent these challenges, if necessary. Their dependence on translation is, in other words, not impenetrable. This is not the case for monolinguals, and they thereby engage with a text in a foreign language at an inevitable increased epistemic risk. This risk, and its inevitability, is shared by all instances of indirect epistemic dependence; also where the translated scientific labor is the findings of a scientific (sub)discipline rather than a text. Here, translational choices might take the form of questions of interpretation-for instance concerning data or a mathematical formalism - and issues of intertranslatability are for instance very vividly captured by the challenge of moving between a mathematical formalism and natural language. Again, with sufficient interactional expertise to acquire the findings from their original context, one can circumvent these challenges, if necessary. ${ }^{23}$ However, those without

\footnotetext{
22 This is not to say that impenetrable epistemic dependence cannot occur outside of broad interdisciplinarity, only that the epistemic circumstances in broad interdisciplinarity render such collaborations susceptible to impenetrable epistemic dependence. Having considered only two case studies here, it remains an open question exactly how widespread impenetrable epistemic dependence is in broad interdisciplinarity and elsewhere. Thank you to an anonymous reviewer of Journal of General Philosophy of Science for raising this issue.

${ }^{23}$ Even in the original context these challenges might re-occur if the reporting of research in the form of scientific publications already qualifies as a translation. We shall ignore this issue here since direct, translucent epistemic dependence is not the focus of discussion.
} 
such interactional expertise-for instance those with mono-disciplinary training engaging in broad interdisciplinarity-face an inevitable increased epistemic risk due to the reliance on translations.

Treating work such as Barad's as generic translations between languages, however, does not exhaust the epistemic challenges to impenetrable epistemic dependence since it generally fails to capture opaqueness. From the translation of a piece of (written) philosophical labor, for instance, one is typically able to assess the argument and thus the findings of the text; under the appropriate consideration of i-iii. Translations between languages-in the narrow sense-do not typically preclude content assessment. This is in contrast to cases of impenetrable epistemic dependence and thus the epistemic circumstances that obtain when Kirby relies on Barad's translation from quantum mechanics. An analogy to the translation of poetry can expose some of these additional epistemic risks. For purposes of illustration, imagine a monolingual English speaker who is interested in assessing the quality of a Japanese haiku. This monolingual might consult a word for word translation, but this will arguably not do for such an assessment, and neither will a retelling of the poem in English. The original context, including the syntax, script, broader cultural embedding, etc., will be crucial for the assessment of the Japanese haiku. The translation could feature remarks about these aspects and perhaps even the translator's and others' assessment of the quality of the haiku, but none of them will bring the monolingual in a position to personally assess the quality of the poem. Irrespective of how faithful the translation is, the relation between the monolingual and the Japanese haiku remains opaque. The monolingual will depend on the translator to acquire the haiku and on the translator's assessment of it. This is the condition also shared under impenetrable epistemic dependence with the quality of the poem replaced by the credibility of the piece of scientific labor relied upon. Without sufficient interactional expertise, no translation-irrespective of how faithful it is-will allow its reader to personally assess the findings. This is the sense in which this type of epistemic dependence is not only opaque but impenetrable: There is no way through the translator.

This is not the same as saying that the epistemic circumstances are fixed. The next section will discuss initiatives that might lessen (but not eliminate!) the epistemic risks involved in depending impenetrably on a translation. First, however, the hazards of interdisciplinary borrowing as they apply to Barad's agential realism will serve as an example of how the risks relating to interlanguage translations generalize to translations between disciplines, and how they are amplified by impenetrable epistemic dependence.

Julie Klein lists six hazards or vulnerabilities that generally obtain in interdisciplinary borrowing:

1. distortion and misunderstanding of borrowed material

2. use of data, methods, concept, and theories out of context

3. use of borrowing out of favor in their original context [...]

4. "illusions of certainty" about phenomena treated with caution or skepticism in their original disciplines

5. overreliance on one particular theory or perspective; and

6. a tendency to dismiss contradictory tests, evidence, and explanations $(1990,88)$

When the translator borrows findings from a discipline, these are, in other words, among the elements that might affect how these findings are reported in the translation. ${ }^{24}$ For

\footnotetext{
${ }^{24}$ The three points raised in relation to interlanguage translation might be placed as follows: $\mathrm{i}$ (translational choices) in 1 and 5, ii (idiosyncrasies) in 5, and iii (intertranslatability) in 1.
} 
illustration, the worries raised in relation to the affective turn distribute as follows: Mistranslation falls under 1 and 2, the worry that the borrowed material is outdated falls under 3 , reliance on few scientists falls under 5 and overlooking that their work is contested falls under 4 and 6.

When it comes to Barad's work, several of these hazards appear relevant as well. Many of these hazards spring from Barad's overreliance on, and idiosyncratic interpretation of, Bohr's work. Relating to 1-distortion of borrowed material in particular-Barad offers a distinctly ontological account of Bohr's interpretation, which, as Barad also recognizes, differs from the epistemological voice found in Bohr's own account. However, Barad merely describes this as "[d]rawing out the ontological dimensions of Bohr's framework" $(2007,174) .{ }^{25}$ Indeed, Barad observes that she is "not alone in arguing that Bohr's views are more accurately described in realist than antirealist terms" $(2007,317)$ and remarks that "philosopher Henry Folse and I have been the strongest proponents of the minority view that sees Bohr as a realist" $(2007,122)$. According to Barad's realist construal, "Bohr rejects the atomistic metaphysics that takes 'things' as ontologically basic entities" (2003, 138) and elsewhere qualifies that this, for Bohr, includes the rejection of entity realism (2007, 55-56). However, according to Folse, Bohr is a realist precisely because Bohr is an entity realist (Folse 1986; 1994). This indicates that Barad's interpretation of Bohr is peculiar even compared to other realist interpretations, though this is not the place to offer a more detailed argument to this effect. ${ }^{26}$ Rather, it is an occasion to raise a worry of type 1 that Barad distorts borrowed material in the form of Bohr's interpretation to her own purposes. $^{27}$

Hollin et al. (2017) raise a concern about Barad's work that falls under 2: use out of context. They worry "about the applicability of concepts originating in the quantum realm and what is lost when they 'jump scales' and are used in order to grasp macro-sociological concerns" $(2017,91)$. Though the authors do not develop this criticism in further detail, it seems to take the form of a worry about the use of quantum concepts away from their typical field of application. Pinch, with his remark "although physicists often claim some sort of allegiance to the Copenhagen Interpretation of quantum mechanics they often do not in fact to subscribe to Bohr's" $(2011,437)$, seems to raise a worry of type 3: Bohr's interpretation is out of favor in contemporary quantum mechanics.

Both of these form epistemic concerns relating to Barad's borrowing from quantum mechanics that require careful treatment, and which call for increased awareness when relying on Barad's account. When these aspects feature in a translation, they are at risk of being uncritically transmitted to the entire interdisciplinary field that depends on this translation and whose impression of quantum mechanics thereby is distorted.

\footnotetext{
${ }^{25}$ Referring to Bohr, Barad elsewhere explains: "I have mined his writings for his implicit ontological views and have elaborated on them in the development of an agential realist ontology" (Barad 2003, 814).

${ }^{26}$ Generally, any ontological reading of Bohr has been strongly opposed by Bohr's former assistant Aage Petersen (1963) and this view is corroborated by the many non-ontological readings of Bohr which include: epistemic (e.g. Murdoch 1987), pragmatist (e.g. Stapp (1972) and Folse (2017)), functionalist (e.g. Camilleri and Schlosshauer 2015), Kantian (e.g. Honner 1987), instrumentalist (e.g. Popper 1962, Ch. 3), and naturalist (e.g. Faye 2017) readings of Bohr. There are ontological readings of Bohr (e.g. Favrholdt 1994; Zinkernagel 2016) but even among these, Barad's reading of Bohr stands out. Like Barad, some authors propose a relational holism implicit in Bohr's writings (e.g. Faye 1991; Dorato 2017), but they emphasize the epistemic character of the holism.

27 This worry relating to Barad resembles an issue that Papoulias and Callard raise in the context of affect theory of which they write: "This conceptualization of affect involves, we have argued, significant distortions of the neurobiological and developmental psychological literature" $(2010,47)$.
} 
This is even more compromising, since Barad's borrowing from quantum mechanics is liability to 5 (the overreliance on one perspective) and 6 (dismissal of contradictory explanations). These aspects already seem relevant when Barad puts such an emphasis on Bohr's interpretation of quantum mechanics. Barad (2007, 287) mentions in passing the existence of other contending interpretations such as the Everett (many-worlds) interpretation, Bohmian mechanics, or GRW collapse theory. However, they are never elaborated upon. Even other Copenhagen-type interpretations ${ }^{28}$ are only discussed in their exposition by Heisenberg. Similarly, Pinch observes how "Barad also notes that there are views in the philosophy of quantum mechanics that are quite close to her own" ${ }^{29}$ and remarks that it is "puzzling that she never elaborates upon, confronts, or teases out in her text these similarities and differences" (Pinch 2011, 439). The absence of anything but passing mentions of both competing and supporting alternative interpretations ${ }^{30}$ amplifies the one-sidedness of Barad's reliance on Bohr (point 5) and silences voices that might contest agential realism which tends towards point 6 (though it is arguably short of dismissing these alternative conceptions). Relatedly, tendencies towards point 4 can be found in Barad's discussion of quantum experiments - quantum eraser experiments in particular-that reads as though these experiments provide experimental evidence for agential realism: "the quantum eraser experiment confirms Bohr's central point that the objects and the agencies of observation are inseparable parts of a single phenomenon" (Barad 2007, 315). These experiments are of course known to the other interpretations of quantum mechanics and can be dealt with even in Bohmian mechanics (Hiley and Callaghan 2006; for a general treatment, see Egg 2013). Barad does not even mention these alternative accounts of the experiment whereby her presentation is liable to give the "illusions of certainty" of point 4 .

More can (and probably should) be said about the extent of these hazards of interdisciplinary borrowing in Barad's work. However, for present purposes the above suffices to indicate how the presence of these hazards in translations generally affect impenetrable epistemic dependence; again Kirby will serve as the example. Lacking sufficient interactional expertise, Kirby has to rely on a translator's testimony to acquire the findings of quantum mechanics. And since Kirby's epistemic dependence on the translator is impenetrable, Kirby cannot assess these findings either, but must also rely on translators for this purpose. Since Barad is Kirby's sole resource for questions of quantum mechanics, i.e. Barad is the only translator called upon, it is not surprising that Kirby simply assumes Barad's account, including Barad's exposition of the quantum eraser that features centrally in Quantum Anthropologies. However, the result of this is that Kirby takes over all the questionable aspects of Barad's borrowing from quantum mechanics. Kirby acquires a skewed version of the findings of quantum mechanics from Barad, but not only that: Since the epistemic dependence is opaque as well as indirect, Barad's translation also doubles as the basis for Kirby's credence in the testimony provided by the translation. The consequences of this are exemplified by passages quoted earlier where Kirby on Barad's authority claims that "a reality made up of individual objects $[\ldots]$ fails" $(2011,126)$ in quantum

\footnotetext{
${ }^{28}$ Whether Bohr's interpretation of quantum mechanics should even be categorized as a variant of the Copenhagen interpretation or a type of interpretation on its own is disputed. See Faye (1991) for a discussion.

29 These include Mermin's Ithaca interpretation and Rovelli’s relational quantum mechanics (Barad 2007, 332-333).

30 Barad (2007, Ch. 7) does provide a rather detailed account of Bell's inequalities and their consequences for local hidden variable theories, however, none of the current major contending interpretations of quantum mechanics are local hidden variables theories.
} 
mechanics. Bohmian mechanics, however, achieves an ontology of separable individual objects in quantum mechanics at the price of non-locality (see for instance Esfeld et al. 2013). Thus, it remains at least an open question, for instance, whether a metaphysics of individuality is consistent with quantum mechanics contrary to what is reflected in Kirby's remarks based on Barad's authority. Kirby appears oblivious to these important (and controversial) commitments of her thesis that transpire from to her sole reliance on Barad. ${ }^{31}$ In this sense, Barad can be conceived as the translator that makes the inevitably interpretational choices according to a very particular reading of the original text, and Kirby is a monolingual who unknowingly inherits these idiosyncrasies (though in good faith). In relying solely on Barad's authority, Kirby inherits Barad's peculiar interpretive stance with respect to quantum mechanics, while presenting it as though it is the uncontested facts about quantum mechanics. ${ }^{32}$

The point, however, is not to disclose issues in this particular case study. Rather, this case is meant to illustrate a general epistemic pattern that impenetrable epistemic dependence is prone to generate. For Kirby, already the need for a translation is the origin of her arguably precarious epistemic circumstance. Kirby's engagement with quantum mechanics requires a translation, and this engagement is thereby inevitably at the mercy of the character and quality of the translation. This is not to say that all translations have as many worrying features as Barad's. Rather, the issue is that the user of the translation-due to the impenetrable epistemic dependence - cannot know whether the translation is faithful or not. There is, in other words, an inherent epistemic risk related to all instances where the use of translators is inevitable. This has nothing to do with the specifics of Barad's or Kirby's work. Rather, this risk is generated by the pattern of epistemic dependence whereby one part is impenetrably epistemically dependent on another. Section 3 argued that the circumstances in broad interdisciplinarity are such that few will have interactional expertise, and many will therefore be in need of translators. The risks involved in the inevitable use of translators are therefore not peculiar to the borrowing of quantum mechanics into critical theory or, for that matter, to the borrowing of neuroscience in the affective turn. Rather, the type of epistemic circumstance that Kirby exemplifies will likely be widespread in broad interdisciplinarity, though its severity will of course depend on the individual translations. The point, therefore, is ultimately this: If this unfortunate pattern of epistemic dependence is a likely consequence of borrowings/collaborations among disciplines with wide cognitive divergence, then general measures should be taken to manage the associated epistemic risks; especially considering the current call for more and wider interdisciplinarity.

\section{Managing Impenetrable Epistemic Dependence}

In the case of a monolingual researcher who relies on translations of important texts, there are (at least) two initiatives that can help the monolingual become aware of idiosyncratic elements of a translation: The monolingual could use many different translations of the

\footnotetext{
31 A similar pattern is noticed by Fitzgerald and Callard in the context of the affective turn where "the 'ebullient' mode tends to take experimental results and theoretical statements from the neurosciences as more-or-less true - with little contest or context, and in the absence of a sense of the wider, often fierce, epistemological and ontological debates within those sciences" (Fitzgerald and Callard 2014, 11).

32 Kirby does express some sensitivity to this issue, when she writes: "[...] the complexities of the scientific theory surely exceed our disciplinary expertise" (Kirby 2011, 78).
} 
same text or the translation used could be well annotated to indicate where translational choices were made (possibly including what alternatives were considered).

Relying on many translations would distribute the epistemic dependence. Though the epistemic dependence on each translator would remain impenetrable, looking for similarities and differences between several translations could help to inform one's credence in the testimony of each translator. If particular translations have been subject to criticism or appraisal, such assessment could also be included. As explored in further detail by Hardwig, one can have good reasons for a belief "which does not constitute evidence for the truth of the proposition", but merely certifies that "others have good reasons to believe it" $(1985,336)$. A survey assessment on the level of translations could precisely yield such non-truth constitutive evidence that might inform whether a translation is faithful or not. One would still have to rely on translators to both acquire and assess findings- the epistemic dependence remains impenetrable — but with multiple translators offering translations (and perhaps discussing others' translations), one can rely on different translators when acquiring and assessing findings.

This is not so for an annotated translation where translational choices are highlighted and elaborated upon. In this case, the annotations just introduce yet another component to the translation where the user will have to rely on the translator's testimony. In addition, the presence of annotations depends on the translator's initiative. The inclusion of annotations is a measure translators can take to alleviate the precarious epistemic circumstance that users of translations find themselves in. In contrast, the users themselves can initiate a survey assessment of a translation (by comparing it to other translations or looking for criticisms of it). Generally, surveying translations therefore makes the users of translations relatively more independent; they can take their precarious epistemic situation into their own hands. It may therefore appear surprising that the recommendation of this paper is that translators must annotate their translations by emphasizing whenever their translation features elements that are contested in the original context. The reason, however, is that even a survey assessment on the level of translations is likely to be ineffective in broad interdisciplinarity as will be argued in the remainder of this section. In a nutshell, the problem is that the wide cognitive divergence in broad interdisciplinarity entails that alternative translations will be scarce, and the same goes for criticisms or appraisals of the translations that actually exist. There are several interrelated reasons for this which are listed here and discussed in turn below: translations require multi-disciplinary expertise, few can scrutinize translations, and no defined community of researchers are compelled to scrutinize translations.

According to Collins et al. (2007) interactional expertise involves the linguistic socializations into the form of life of a disciplinary community. By this characterization, the mediation between two disciplines involves moving between two languages; interactional expertise provides for a fractionated trading zone. For a third discipline to enter the trading zone, interactional expertise in the language of that discipline is required. The practitioners of the third discipline will have no, or at least very little, benefit from eavesdropping on the communication between the two first disciplines. A translation-as the term has been used here-is the reporting of the findings of a discipline for those who lack the interactional expertise to borrow the findings directly. As such, it also partakes in a fractionated trading zone and by this reasoning, translations are not only from the language of a discipline, but also into the language of another. Those of a third discipline will benefit little from that translation, just like a monolingual English speaker will have very limited use of a translation of Kierkegaard from Danish to German. Following the construal of Collins et al. (2007), those of the third discipline will require a translation of their own and the making 
of a translation therefore requires interactional expertise both in the originating and target discipline. Translations require multi-disciplinary expertise and particularly in broad interdisciplinarity, such expertise is hard to come by. Therefore, few will be in a position to offer a translation, for instance between quantum mechanics and critical theory. ${ }^{33}$ While there are certainly many translations from quantum mechanics, those with expertise in critical theory cannot generally use these-say a translation for mathematicians-for the purpose of a survey assessment among translations. Since they also require expertise in the discipline translated to, these other translations will, by the argument above, be inaccessible in the same way (though perhaps to a different degree) that quantum mechanics is in its original context.

One might overcome this if the few available translations were systematically scrutinized such that potential mistranslations could be disclosed. However, also such a scrutiny of a translation would require relevant multi-disciplinary expertise, though perhaps to a lesser degree than that required to make a translation in the first place. In the context of Barad's work, Michael Lynch writes of this challenge that "it seems necessary to pass through Bohr, quantum physics, and feminist epistemology before engaging directly with Barad's philosophy" $(2014,139)$. One might add the need to pass through several aspects of feminist science studies to this list. As such, any critical scrutiny of Barad's translation would, as Lynch writes, "be quite an undertaking for someone with a limited background in the relevant fields" $(2014,140)$. This is not to say that no-one could undertake such work. However, they will not be numerous due to the need of multi-disciplinary expertise in so cognitively divergent fields as quantum mechanics and critical theory (broadly construed); a state that should generalize to all translations in broad interdisciplinarity.

Finally, translations are subject to typical problems relating to interdisciplinary work. Interdisciplinary work is often marginalized compared to the disciplinary mainstream: "The majority of people engaged in interdisciplinary work lack a common identity. As a result, they often find themselves homeless, in a state of social and intellectual marginality" (Klein 1990, 13). This lack of common identity entails that translators rarely form pseudodisciplinary communities in which the translations are discussed. As Hanne Andersen observes, a consequence of this is that "when a research activity involves multiple disciplines, there is not a well-defined community of practitioners who are all expected to be equally capable of critically scrutinizing new results" (2016, 7). This effect, she argues, is only amplified in broad interdisciplinarity. Because there is no well-defined community, no group of researchers is compelled to scrutinize translations out of a responsibility to the community. As such, even those who might have the expertise to scrutinize translations rarely do so. Thus, criticisms and appraisals of translations cannot be expected to be frequent either, and their existence can therefore not be assumed in broad interdisciplinarity whereby survey assessments of the translations are in general ineffective. Survey assessments of translations can therefore not be what is generally relied upon to secure the epistemic integrity in cases of impenetrable epistemic dependence.

The general absence of critical scrutiny of translations is well illustrated by the case of Barad. The literature abounds with discussions and criticisms of the critical theory components of Barad's work. However, when Kirby relies on Barad as translator, these discussions of Barad's contributions to critical theory are irrelevant for Kirby's assessment as to whether Barad's translation is faithful. Unfortunately, and as expected given its character

\footnotetext{
${ }^{33} \mathrm{We}$ shall here set aside the otherwise relevant question exactly when two communities are different enough to have different languages. The reader is referred to Collins et al. (2007, Sect. 2.3) for a discussion.
} 
as broad interdisciplinarity, a survey of the thousands of citations of Meeting the Universe Halfway reveals few that discuss the relation between quantum mechanics and critical theory and even fewer that discuss the translation from quantum mechanics itself. Though for instance Harrell (2016), in her exploration of feminist philosophy of physics, provides an account of Barad's agential realist interpretation of quantum mechanics, the legitimacy of this interpretation is never discussed and neither is it assessed whether it connects with the critical theory conclusions drawn from it. Hollin et al. (2017), as already mentioned, go a bit further when they worry about the use of agential realism in the macroscopic domain. However, the authors merely raise the question, mention that physicists find such a jump problematic, but do not even substantiate what is at issue when going from micro- to macro-scale. Pinch's (2011) remarks about the existence of other interpretations of quantum mechanics are similarly limited to raising the issue without further substantiation. The latter two explicitly recognize that they lack the interactional expertise to develop these criticisms in further details which corroborates the claim that such interactional expertise is hard to come by.

The marginalization of translations is also illustrated by the striking absence of any mention of Barad's work in the anthology Niels Bohr and the Philosophy of Physics: Twenty-First-Century Perspectives (Faye and Folse 2017). Despite being beyond doubt the most influential interpreter of Bohr as measured by the number of citations, Barad's interpretation is nested in a translation of quantum mechanics into critical theory that marginalizes it within the community of interpreters of Bohr. Though these scholars might anyway lack the relevant expertise in critical theory to profoundly scrutinize Barad's translation, being often philosophers of physics by training they would possibly be able to assess the content relating to Bohr's interpretation of quantum mechanics. The same might be the case for philosophers of quantum mechanics. However, neither community have been compelled to discuss Barad's work whereby, again, the works of criticism and appraisal needed for a survey assessment of the translation is absent.

\section{Towards a Solution}

Epistemic dependence is most often approached from the side of the dependent part: what conditions must one meet to be rationally epistemically dependent ${ }^{34}$ But as already argued, impenetrable epistemic dependence constitutes a circumstance where even nontruth constitutive reasons for a proposition-reasons not constituting first order evidence for the proposition - are hard to come by; again, due to the absence of alternative translations or other works that critically scrutinize the existing translations. ${ }^{35}$ This significantly reduces the prospects for bettering the epistemic conditions occurring in cases of impenetrable epistemic dependence by the introduction of epistemic norms of belief on the dependent part. The only immediate alternative is a testifier perspective that introduces additional epistemic norms of assertion on the part of the testifier, what will be denoted norms of testimony below.

\footnotetext{
${ }_{34}$ Often following Hardwig (1985) and Goldman (2001). See Pritchard (2015) for a recent review of this perspective.

35 This raises the question whether a proposition can be rationally held on the basis of impenetrable epistemic dependence even if we follow Hardwig and acknowledge that non-truth constitutive reasons can count towards rational belief.
} 
A number of competing frameworks are on the table when it comes to norms of assertion: the knowledge norms of assertion (e.g. Williamson 2000), the reasonable to believe norm of assertion (e.g. Lackey 2007), truth norms of assertion (e.g. Weiner 2005), to name some. But only Gerken's (2012) warrant-assertive speech act (WASA) norm has been directly applied to testimony in intra-scientific collaboration (Gerken 2015), and we shall therefore focus on it here. More precisely, Gerken suggests that a variant of WASA, the discursive justification-assertion (DJA) account for conversational contexts, should apply to intra-scientific testimony:

In the discursive conversational context, DCC, in which S's assertion that $p$ conveys that $p, \mathrm{~S}$ meets the epistemic conditions on appropriate assertion that $p$ (if and) only if S's assertion is appropriately based on a degree of discursive justification for believing that $p$ that is adequate relative to DCC (Gerken 2015, 576).

DJA captures the intuition that the testifier should abide by certain norms that take the form of an ability to articulate - qua discursive-evidence for the testimony. In addition, it introduces a gradeability that might capture the special circumstances occurring in impenetrable epistemic dependence: "the degree of discursive justification [...] depends on the specific context in which the intra-scientific testimony takes place" (Gerken 2015, 579, emphasis in original). However, applying this norm to the case study indicates that issues of justification are largely orthogonal to the problems raised above about Barad's borrowing from quantum mechanics. While DJA is arguably a necessary norm for broad interdisciplinarity, it is not sufficient.

In essence, Barad's Meeting the Universe Halfway is a tour de force in justification for agential realism. If the degree of justification is inappropriate, it is because Barad's overdoes it—violating a Gricean maxim of quantity — considering that much of the likely audience has no interactional expertise in physics and therefore has little appreciation for the details that Barad provides. Barad's justification includes careful discussions of the workings of quantum mechanics in both real and thought experiments (Barad 2007, Ch. 7). Throughout her book, Barad also offers extensive textual evidence for her reading of Bohr. The relevance of agential realism beyond quantum mechanics is also discussed in several places (e.g. Barad 2007, 275-280, 323-327). Here Barad promotes the view that "the overwhelming empirical success of quantum theory suggests that it is a theory that supersedes Newtonian physics" (Barad 2007, 324, emphasis in original). The point apparently being that since the true description of reality is obtained with quantum mechanics and not Newtonian physics, the quantum metaphysics is the true metaphysics: "there are not two separate domains of nature, one macroscopic and one microscopic" (Barad 2007, 338). Barad does, in other words, provide justification for agential realism and even for the extension of this quantum metaphysics beyond the usual context; the lack of justification is not the problem in Barad's work.

Rather, the problem is that Barad's interpretation of quantum mechanics is idiosyncratic or at least only one interpretation among many; something that one could immediately realize upon a comparison to the rest of the literature on the interpretation of quantum mechanics. However, this was exactly the key why this is a particular issue obtaining in instances of impenetrable epistemic dependence: those whose epistemic dependence on Barad is impenetrable are restrained from this comparison. They can only access the translation provided by Barad, her testimony, and thus receive only her conclusions along with the (largely inaccessible) justifications provided for them. Though the literature on the interpretation of quantum mechanics, as argued, does not directly engage with Barad's writing, the availability of a number of significantly different contending interpretations of 
quantum mechanics such as Bohmian mechanics discussed above implicitly raises questions whether Barad's conclusions are warranted by her justification or at least suggests that they are underdetermined by the evidence. This, however, is lost on those whose lack of interactional expertise renders them impenetrably epistemically dependent on a translator; as exemplified by Kirby's reliance on Barad. Disputes and disagreements are only available if they are translated or if there exists a community of translators that can critically scrutinize the translation. The absence of a treatment of the other interpretations of quantum mechanics by Barad herself and the lack of a proper community of translators between quantum mechanics and critical theory have the consequence that the deep disagreements about the interpretation of quantum mechanics are never available to those without the interactional expertise to borrow directly from quantum mechanics. This is how Kirby, for instance, unknowingly comes to commit to a particular-and at places very idiosyncratic-interpretation of quantum mechanics and mistakenly comes to state it as fact.

In so far as this condition, as already argued, is typical of broad interdisciplinarity, it seems that additional instruments beyond DJA must be in place to secure the epistemic integrity of broad interdisciplinarity where not all can be assumed to have (sufficient) interactional expertise due to cognitive divergence. One may argue that DJA already does the job: that the above just demonstrates how Barad's justification is contentious which in turn could imply that the degree of discursive justification is insufficient in Barad's translation from quantum mechanics to critical theory; it thus indirectly connects back to justification and therefore into the scope of DJA. But I think it is instructive to cast this as a special problem in the context of translations and impenetrable epistemic dependence beyond the translators' justification and therefore beyond the scope of DJA. In other words, it is instructive to think that special norms of testimony beyond appropriate justification apply in broad interdisciplinarity for those acting as translators. I propose that translators must assume that their translations will largely escape critical scrutiny (for the reasons given in Sect. 5) and that parts of their readership can only acquire and assess the translation by means of the translation itself. This emphasizes that acting as a translator offering translation is a special role compared to other roles in scientific collaboration which includes that of acting as a fellow scientist offering a point of view or acting as an expert providing testimony to collaborators with enough interactional expertise to avoid impenetrable epistemic dependence. This special role as a translator calls for special norms of testimony.

Following the analogy of annotated inter-language translations, I propose that the special commitment of translators (SCT) beyond that of other experts is this:

SCT: (a) that all testimony in broad interdisciplinarity must aim to be neutral with respect to disputed issues within the relevant disciplines and (b) that any deviation from (a) must be clearly highlighted. ${ }^{36}$

This resolves the catch-22 of broad interdisciplinarity: that those without interactional expertise have to rely on translators, but that translators-due to the cognitive divergence in broad interdisciplinarity - are few whereby even a survey assessment of the translation is often challenged. With SCT, even those without sufficient interactional expertise have means to ascertain their level of confidence in the conclusions of the translation. This norm can of course not work miracles. It cannot resolve all the issues with impenetrable

\footnotetext{
36 The norm is neutral with respect to the question whether it is possible to report TRUTH in translations. Whether a proposition is contested is entirely a question of how it is perceived by the community of researchers in its original context.
} 
epistemic dependence; it is still a very precarious epistemic circumstance. One concern might be that unreliable translators are also unreliable testifiers about the neutrality of their translations. Obviously, this is beyond the scope of SCT to remedy, but it is also not the point. Take the example of Barad: she is not an unreliable translator (as testified by the amount of justification she gives) and nothing indicates that she is unaware that her account is conflicting with other serious interpretations of quantum mechanics. Had Barad aimed to comply with SCT, we have no reason to believe that she would not do this reliably. Norms cannot be a bulwark against questionable research practice-for instance in the form of unreliable translation-but since most aim at a responsible conduct of research, promoting SCT to an epistemic norm in broad interdisciplinarity ensures that we can expect it to be adhered to in the same way as other norms of science. Thus, we should trust translators' reports about their compliance to SCT, i.e. their reporting on the neutrality of their translation. SCT is meant to take care of an issue in broad interdisciplinarity that occurs, not because of scientific misconduct, but as a result of the peculiar conditions obtaining in impenetrable epistemic dependence. It is meant to secure that contentious elements of translations are clearly highlighted to avoid that entire subfields build on a questionable foundation without knowing about it.

\section{Conclusion}

While much interdisciplinarity brings together proximate fields, this is not always the case. Broad interdisciplinarity sees integration between disciplines that are perceived to be nonneighboring and disparate fields. This paper has argued that the heterogeneity among disciplines in broad interdisciplinarity calls for stricter epistemic norms of testimony for experts that act as translators between the disciplines than those suggested for intra-scientific testimony. This view was developed from a study of Barad's agential realism and its use in critical theory, particularly in Kirby's Quantum Anthropologies. Kirby holds no interactional expertise in quantum mechanics and must therefore rely on translations of quantum mechanics to critical theory - such as that provided by Barad - to engage in this interdisciplinary field. Kirby — and others with insufficient interactional expertise- - was argued to be in a condition of impenetrable epistemic dependence; a case of opaque, indirect epistemic dependence where one is unable to assess the evidence for the conclusions one depends on and where the conclusions are acquired from a translator and not directly from their original context. While Barad most plausibly abides by usual norms of testimony relating to justificatory requirements (such as Gerken's DJA), she still offers a very idiosyncratic interpretation of quantum mechanics as disclosed by a comparison with the treatment of quantum mechanics within the philosophy of physics. However, dealing only with this other literature in her endnotes and not very extensively, Barad's text largely conveys an impression that she is simply explicating the "epistemological and ontological issues that quantum physics forces us to confront" (Barad 2007, 24). Providing this as expert testimony for the broad interdisciplinary integration between quantum mechanics and critical theory, those who rely on this testimony unknowingly commit to a disputed interpretation, as exemplified by Kirby's Quantum Anthropologies. Consequently, the epistemic credentials of this testimony are questionable, and due to the condition of impenetrable epistemic dependence these epistemic issues are unknowingly inherited by those who-like Kirbyrely on this testimony. Barad's conduct is arguably defensible if she engaged within a community such as philosophy of quantum mechanics where her account would be subject to 
criticism. However, since her work (primarily) takes the form of a translation for researchers in another discipline, it escapes the eyes of those who might have expertise in the discipline translated from; and who might anyway find the work inaccessible since it translates into critical theory. Barad's and other translations therefore largely escape critical scrutiny. Consequently, it was argued that additional norms of testimony should be adhered to by translators in broad interdisciplinarity (SCT): (a) all testimony in broad interdisciplinary must be neutral with respect to disputed issues within relevant disciplines and (b) any deviation from (a) must be clearly highlighted. This is meant to improve the accessibility of the credentials of the testimony even to those in a condition of impenetrable epistemic dependence. Any translation that deviates from SCT risks the epistemic integrity of the field that, out of necessity, relies on its testimony. Translations are an indispensable, but also largely unchecked type of interdisciplinary contribution and therefore special norms of testimony should apply to translations.

Acknowledgements I would like to express my gratitude to Hanne Andersen, Astrid Rasch, Sebastian Renstrøm, Kian Salimkani, Steve Fuller, Vivian Anette Lagesen, and two anonymous reviewers of Journal for General Philosophy of Science for their valuable feedback on and helpful discussion of earlier drafts of this paper.

Funding Open Access funding provided by NTNU Norwegian University of Science and Technology (incl St. Olavs Hospital - Trondheim University Hospital).

Open Access This article is licensed under a Creative Commons Attribution 4.0 International License, which permits use, sharing, adaptation, distribution and reproduction in any medium or format, as long as you give appropriate credit to the original author(s) and the source, provide a link to the Creative Commons licence, and indicate if changes were made. The images or other third party material in this article are included in the article's Creative Commons licence, unless indicated otherwise in a credit line to the material. If material is not included in the article's Creative Commons licence and your intended use is not permitted by statutory regulation or exceeds the permitted use, you will need to obtain permission directly from the copyright holder. To view a copy of this licence, visit http://creativecommons.org/licenses/by/4.0/.

\section{References}

Andersen, H. (2016). Collaboration, interdisciplinarity, and the epistemology of contemporary science. Studies in History and Philosophy of Science Part A, 56, 1-10. https://doi.org/10.1016/j.shpsa .2015.10.006.

Aronowitz, S. (1988). Science as power: Discourse and ideology in modern society. Minneapolis: University of Minnesota Press.

Aspect, A., Grangier, P., \& Roger, G. (1982). Experimental realization of Einstein-Podolsky-Rosen-Bohm Gedankenexperiment: A new violation of Bell's inequalities. Physical Review Letters, 49(2), 91-94.

Barad, K. (1984). Minimal lattice theory of fermions. Physical Review D, 30(6), 1305-1309. https://doi. org/10.1103/PhysRevD.30.1305.

Barad, K. (1988). Quenched fermions on the Columbia lattice parallel processor. Nuclear Physics B, Proceedings Supplements, 4(April), 165-169. https://doi.org/10.1016/0920-5632(88)90096-5.

Barad, K. (2003). Posthumanist performativity: Toward an understanding of how matter comes to matter. Signs Journal of Women in Culture and Society, 28(3), 801-831.

Barad, K. (2007). Meeting the universe halfway. Durham: Duke University Press.

Barad, K. (2011). Erasers and erasures: Pinch's unfortunate 'uncertainty principle'. Social Studies of Science, 41(3), 443-454. https://doi.org/10.1177/0306312711406317.

Barad, K. (2012). On touching-The inhuman that therefore I am. Differences, 23(3), 206-223. https://doi. org/10.1215/10407391-1892943.

Bell, J. S. (1964). On the Einstein Podolsky Rosen paradox. Physics Physique Fizika, 1(3), 195-200.

Bowman, N. (2019). Here/there/everywhere: Quantum models for decolonizing Canadian state onto-epistemology. Foundations of Science. https://doi.org/10.1007/s10699-019-09610-x. 
Camilleri, K., \& Schlosshauer, M. (2015). Niels Bohr as philosopher of experiment: Does decoherence theory challenge Bohr's doctrine of classical concepts? Studies in History and Philosophy of Science Part B: Studies in History and Philosophy of Modern Physics, 49, 73-83.

Clough, P. T., \& Halley, J. (2007). The affective turn: Theorizing the social. Durham: Duke University Press.

Coleman, R. (2014). Inventive feminist theory: Representation, materiality and intensive time. Women: A Cultural Review, 25(1), 27-45. https://doi.org/10.1080/09574042.2014.901098.

Collins, H., \& Evans, R. (2007). Rethinking expertise. Chicago, IL: Chicago University Press.

Collins, H., Evans, R., \& Gorman, M. (2007). Trading zones and interactional expertise. Case Studies of Expertise and Experience, 38(4), 657-666. https://doi.org/10.1016/j.shpsa.2007.09.003.

Damasio, A. (2003). Looking for Spinoza: Joy, sorrow and the feeling brain. London: William Heinemann.

Dolphijn, R., \& Van der Tuin, I. (2012). New materialism: Interviews \& cartographies. New Metaphysics: Open Humanities Press. https://doi.org/10.3998/ohp.11515701.0001.001.

Dorato, M. (2017). Bohr's relational holism and the classical-quantum interaction. In J. Faye \& H. J. Folse (Eds.), Niels Bohr and the philosophy of physics: Twenty-first-century perspectives (pp. 133-154). London: Bloomsbury Academic.

Dunk, R. A. (2019). Diffracting the 'quantum' and the 'social': Meeting the universe halfway in social science. Cultural Studies Critical Methodologies. https://doi.org/10.1177/1532708619880212.

Egg, M. (2013). Delayed-choice experiments and the metaphysics of entanglement. Foundations of Physics, 43(9), 1124-1135. https://doi.org/10.1007/s10701-013-9734-4.

Einstein, A., Podolsky, B., \& Rosen, N. (1935). Can quantum-mechanical description of physical reality be considered complete? Physical Review, 47(10), 777-780. https://doi.org/10.1103/PhysRev.47.777.

Esfeld, M., Hubert, M., Lazarovici, D., \& Dürr, D. (2013). The ontology of Bohmian mechanics. The British Journal for the Philosophy of Science, 65(4), 773-796. https://doi.org/10.1093/bjps/axt019.

Favrholdt, D. (1994). Niels Bohr and realism. In J. Faye \& H. J. Folse (Eds.), Niels Bohr and contemporary philosophy (pp. 77-96). Dordrecht: Springer.

Faye, J. (1991). Niels Bohr: His heritage and legacy, an anti-realist view of quantum mechanics. Dordrecht: Kluwer Academic Publishers.

Faye, J. (2017). Complementarity and human nature. In J. Faye \& H. J. Folse (Eds.), Niels Bohr and the philosophy of physics: Twenty-first-century perspectives (pp. 115-131). London: Bloomsbury Academic.

Faye, J., \& Folse, H. J. (2017). Niels Bohr and the philosophy of physics: Twenty-first-century perspectives. London: Bloomsbury.

Fitzgerald, D., \& Callard, F. (2014). Social science and neuroscience beyond interdisciplinarity: Experimental entanglements. Theory, Culture \& Society, 32(1), 3-32. https://doi.org/10.1177/026327641453731 9.

Folse, H. J. (1986). Niels Bohr, complementarity, and realism. PSA: Proceedings of the Biennial Meeting of the Philosophy of Science Association, 1986, 96-104.

Folse, H. J. (1994). Bohr's framework of complementarity and the realism debate. In J. Faye \& H. J. Folse (Eds.), Niels Bohr and contemporary philosophy (pp. 119-139). Dordrecht: Springer.

Folse, H. J. (2017). Complementarity and pragmatic epistemology: A comparison of Bohr and C.I Lewis. In J. Faye \& H. J. Folse (Eds.), Niels Bohr and the philosophy of physics: Twenty-first-century perspectives (pp. 91-114). London: Bloomsbury Academic.

Gamble, C. N., Hanan, J. S., \& Nail, T. (2019). What is new materialism? Angelaki, 24(6), 111-134. https:// doi.org/10.1080/0969725X.2019.1684704.

Gerken, M. (2012). Discursive justification and skepticism. Synthese, 189(2), 373-394. https://doi. org/10.1007/s11229-012-0076-0.

Gerken, M. (2015). The epistemic norms of intra-scientific testimony. Philosophy of the Social Sciences, 45(6), 568-595. https://doi.org/10.1177/0048393115600527.

Goldman, A. I. (2001). Experts: Which ones should you trust? Philosophy and Phenomenological Research, 63(1), 85-110. https://doi.org/10.2307/3071090.

Götschel, H. (2011). The entanglement of gender and physics: Human actors, work place cultures, and knowledge production. Science \& Technology Studies, 24(1), 66-80.

Hadač, E. (1977). Complex interdisciplinary investigation of landscape. Landscape Planning, 4(January), 333-348. https://doi.org/10.1016/0304-3924(77)90039-9.

Harding, S. (1986). The science question in feminism. ACLS Humanities E-Book. Ithaca: Cornell University Press.

Harding, S. (1991). Whose science? Whose knowledge?. Ithaca: Cornell University Press.

Hardwig, J. (1985). Epistemic dependence. The Journal of Philosophy, 82(7), 335-349. https://doi. org/10.2307/2026523. 
Harrell, M. (2016). On the possibility of feminist philosophy of physics. In M. C. Amoretti \& N. Vassallo (Eds.), Meta-philosophical reflection on feminist philosophies of science (pp. 15-34). Berlin: Springer. https://doi.org/10.1007/978-3-319-26348-9_2.

Hayles, K. (1984). The cosmic web. Ithaca: Cornell University Press.

Hiley, B. J., \& Callaghan, R. E. (2006). Delayed-choice experiments and the Bohm approach. Physica Scripta, 74(3), 336-348. https://doi.org/10.1088/0031-8949/74/3/007.

Hinton, P. (2017). A sociality of death: Towards a new materialist politics and ethics of life itself. In V. Kirby (Ed.), What if culture was nature all along? (pp. 223-247). Edinburgh: Edinburgh University Press.

Hollin, G., Forsyth, I., Giraud, E., \& Potts, T. (2017). (Dis)Entangling Barad: Materialisms and ethics. Social Studies of Science, 47(6), 918-941. https://doi.org/10.1177/0306312717728344.

Honner, J. (1987). The description of nature: Niels Bohr and the philosophy of quantum physics (Vol. 179). Oxford: Oxford University Press.

Keller, E. F. (1995). Reflections on gender and science. New Haven: Yale University Press.

Kirby, V. (2011). Quantum anthropologies: Life at large. Durham: Duke University Press.

Kitcher, P. (1990). The division of cognitive labor. The Journal of Philosophy, 87(1), 5-22. https://doi. org/10.2307/2026796.

Klein, J. T. (1990). Interdisciplinarity: History, theory, \& practice. Detroit: Wayne State University Press.

Klein, J. T. (2010). A taxonomy of interdisciplinarity. In R. Frodeman, J. T. Klein, \& R. C. D. S. Pacheco (Eds.), The Oxford handbook of interdisciplinarity (1st ed.). Oxford: Oxford University Press.

Krishna, D. (1977). Culture. International Social Science Journal, 29(4), 651-670.

Lackey, J. (2007). Norms of assertion. Noûs, 41(4), 594-626.

Lynch, M. (2014). Matters of fact, and the fact of matter. Human Studies, 37(1), 139-145. https://doi. org/10.1007/s10746-013-9297-6.

Malabou, C. (2012). The new wounded from neurosis to brain damage. Forms of living (1st ed.). New York: Fordham University Press.

Murdoch, D. R. (1987). Niels Bohr's philosophy of physics. Cambridge: Cambridge University Press.

Nadeau, R., \& Kafatos, M. (2001). The non-local universe: The new physics and matters of the mind. Cary: Oxford University Press.

Newell, W. (1998). Professionalizing interdisciplinarity: A literature review and research agenda. In W. Newell (Ed.), Interdisciplinarity: Essays from the literature (pp. 529-563). New York: The College Board.

Papoulias, C., \& Callard, F. (2010). Biology's gift: Interrogating the turn to affect. Body \& Society, 16(1), 29-56. https://doi.org/10.1177/1357034X09355231.

Petersen, A. (1963). The philosophy of Niels Bohr. Bulletin of the Atomic Scientists, 19(7), 8-14.

Pickering, A. (1984). Constructing quarks: A sociological history of particle physics. Edinburgh: Edinburgh University Press.

Pinch, T. (2011). Karen Barad, quantum mechanics, and the paradox of mutual exclusivity. Social Studies of Science, 41(3), 431-441.

Plotnitsky, A. (1994). Complementarity: Anti-epistemology after Bohr and Derrida. Durham: Duke University Press.

Popper, K. R. (1962). Conjectures and refutations: The growth of scientific knowledge. New York: Routledge.

Pritchard, D. (2015). Epistemic dependence. Philosophical Perspectives, 29(1), 305-324. https://doi. org/10.1111/phpe.12067.

Richardson, S. S. (2010). Feminist philosophy of science: History, contributions, and challenges. Synthese, 177(3), 337-362. https://doi.org/10.1007/s11229-010-9791-6.

Rouse, J. (2004). Barad's feminist naturalism. Hypatia, 19(1), 142-161.

Stapp, H. (1972). The Copenhagen interpretation. American Journal of Physics, 40, 1098-1116.

Thagard, P. (1997). Collaborative knowledge. Nous, 31(2), 242-261. https://doi.org/10.1111/00294624.00044.

Turner, M. (1996). The literary mind. New York: Oxford University Press.

Wagenknecht, S. (2014). Opaque and translucent epistemic dependence in collaborative scientific practice. Episteme, 11(4), 475-492. https://doi.org/10.1017/epi.2014.25.

Weiner, M. (2005). Must we know what we say? The Philosophical Review, 114(2), 227-251. https://doi. org/10.1215/00318108-114-2-227.

Weisberg, M., \& Muldoon, R. (2009). Epistemic landscapes and the division of cognitive labor*. Philosophy of Science, 76(2), 225-252. https://doi.org/10.1086/644786.

Wendt, A. (2015). Quantum mind and social science: Unifying physical and social ontology. Cambridge: Cambridge University Press. https://doi.org/10.1017/CBO9781316005163. 
Willey, A. (2016). A world of materialisms: Postcolonial feminist science studies and the new natural. Science, Technology and Human Values, 41(6), 991-1014. https://doi.org/10.1177/0162243916658707.

Williamson, T. (2000). Knowledge and its limits. Oxford: Oxford University Press.

Zinkernagel, H. (2016). Niels Bohr on the wave function and the classical/quantum divide. Studies in History and Philosophy of Science Part B: Studies in History and Philosophy of Modern Physics, 53, 9-19.

Publisher's Note Springer Nature remains neutral with regard to jurisdictional claims in published maps and institutional affiliations. 